\title{
Coulomb Systems Seen as Critical Systems : Ideal Conductor Boundaries
}

\section{B. Jancovici ${ }^{1}$ and G. Téllez ${ }^{1}$}

\begin{abstract}
$\underline{\text { Abstract }}$
The grand potential of a classical Coulomb system has universal finite-size corrections similar to the ones which occur in the free energy of a simple critical system : the massless Gaussian field. Here, the Coulomb system is assumed to be confined by walls made of an ideal conductor material ; this choice corresponds to simple (Dirichlet) boundary conditions for the Gaussian field. For a $d$-dimensional $(d \geq 2)$ Coulomb system confined in a slab of thickness $W$, the grand potential (in units of $k_{B} T$ ) per unit area has the universal term $\Gamma(d / 2) \zeta(d) / 2^{d} \pi^{d / 2} W^{d-1}$. For a two-dimensional Coulomb system confined in a disk of radius $R$, the grand potential (in units of $k_{B} T$ ) has the universal term $(1 / 6) \ln R$. These results, of general validity, are checked on two-dimensional solvable models.
\end{abstract}

KEY WORDS : Critical systems ; finite size effects ; Coulomb systems ; solvable models.

LPTHE Orsay 95-10

March 1995

1 Laboratoire de Physique Théorique et Hautes Energies, Université de Paris-Sud, 91405 Orsay, France (Laboratoire Associé au Centre National de la Recherche Scientifique - URA 63). E-mail : janco, tellez @ stat.th.u-psud.fr 


\section{INTRODUCTION}

A classical Coulomb system is a system of charged particles, interacting through the Coulomb potential, plus perhaps some short-range interaction ; there may also be a continuous charged background. We are interested in equilibrium properties, and classical (i.e. non-quantum) statistical mechanics is used. A Coulomb system may have phase transitions (at least in the two-dimensional case) ; here we assume the system to be in a conducting phase.

In some geometries, it has already been shown ${ }^{(1,2)}$ that the free energy (or the grand potential) of a classical Coulomb system exhibits universal finite-size corrections, in close analogy with what happens in critical systems ${ }^{(3-6)}$. In Coulomb systems, although the screening effect makes the charge correlations short-ranged, that same screening effect makes the electric potential and field correlations long-ranged ${ }^{(7)}$; the critical-like behavior of the free energy is related to the existence of these long-ranged correlations. The electric potential is in some sense the analog of the order parameter of a critical system.

For defining a Coulomb system with boundaries, one has to state the boundary conditions. In a previous paper ${ }^{(2)}$, we considered the case when the boundary is a plain hard wall which confines the charges. Such an assumption does not generate any simple boundary conditions for the electric potential : for a given charge distribution, the potential "leaks out", taking in general non-zero values outside the Coulomb system and that spoils the analogy with the order parameter of a critical system.

The purpose of the present paper is to study classical Coulomb systems with boundaries, in the simpler case of Dirichlet boundary conditions for the electric potential : the walls, impenetrable to the particles of the Coulomb system, are assumed to be made of an ideal conductor material on which the electric potential vanishes. In general, one must also assume that there is some wall-particle short-range repulsion which prevents a particle from collapsing onto its electric image. 
Two geometries will be considered.

(a) Slab. In $d$ dimensions $(d \geq 2)$, a Coulomb system is confined between two parallel ideal conductor plates, separated by a distance $W$; the Coulomb system extends to infinity in the $d-1$ other directions. Let $\omega$ be the grand potential per unit area. We shall show that $\omega$ (times the inverse temperature $\beta$ ) has the large- $W$ expansion

$$
\beta \omega=A W+B+\frac{C(d)}{W^{d-1}}+\cdots
$$

The first two terms represent, respectively, the bulk and the surface contributions ; the coefficients $A$ and $B$ are non-universal. However, the last term of (1.1) is universal, with a coefficient $C(d)$ depending only on the dimension $d$ :

$$
C(d)=\frac{\Gamma\left(\frac{d}{2}\right) \zeta(d)}{2^{d} \pi^{\frac{d}{2}}}
$$

where $\Gamma$ and $\zeta$ are the gamma function and the Riemann zeta function. In particular, $C(2)=\pi / 24$.

Before we derived the expansion (1.1), we guessed it by analogy with a similar expansion which is valid for a simple critical system : the massless Gaussian field theory. We define the partition function of that theory as the functional integral on a field $\phi(\mathbf{r})$

$$
Z_{G}=\int \mathcal{D} \phi(\mathbf{r}) \exp \left[-\frac{\beta}{2 \mu_{d}} \int \phi(\mathbf{r})(-\Delta) \phi(\mathbf{r}) d \mathbf{r}\right]
$$

where $\mu_{2}=2 \pi, \mu_{3}=4 \pi$, and more generally, for $d>2, \mu_{d}=(d-2) 2 \pi^{d / 2} / \Gamma(d / 2)$. Some ultraviolet regularization is needed for avoiding divergences. With Dirichlet boundary conditions for $\phi(\mathbf{r})$ on two parallel plates, it was shown ${ }^{(3)}$ that the free energy associated to $Z_{G}$, in the case $d=2$, has a universal term $-\pi / 24 W$. More generally, we shall show that the expansion of the free energy for the Gaussian field theory is of the form (1.1), except for a change of sign of the universal coefficient $C(d)$. 
(b) Disk. A two-dimensional Coulomb system is confined in a disk of radius $R$. The boundary circle is assumed to be an ideal conductor. For that case, we shall show that the grand potential $\Omega$ has the large- $R$ expansion

$$
\beta \Omega=A R^{2}+B R+\frac{1}{6} \ln R+\cdots
$$

where again the coefficients $A$ and $B$ of the bulk and perimeter contributions are nonuniversal, while the term $(1 / 6) \ln R$ is a universal finite-size correction. Here too, we first guessed (1.4) by analogy with a similar expansion ${ }^{(5,6)}$ which holds ${ }^{2}$ for the Gaussian field theory, except for a change of sign of the universal term.

In two dimensions, there are exactly solvable models of Coulomb systems : the twocomponent plasma and the one-component plasma, at some special temperature. The general expansions (1.1) and (1.4) can be explicitly checked on these models.

The critical system which is related to Coulomb systems is the massless Gaussian field theory ; this relation is discussed in Section 2. Section 3 is about the slab geometry, Section 4 about the disk geometry. The Conclusion suggests some further research.

\section{COULOMB SYSTEMS AND GAUSSIAN FIELD THEORY}

There are many signs that there is some connexion between Coulomb systems and the Gaussian field theory. The Coulomb potential is the inverse of the properly normalized Laplacian $-\left(1 / \mu_{d}\right) \Delta$ which appears in (1.3). If $\phi$ is interpreted as the electric potential, the Hamiltonian in (1.3)

$$
H=\frac{1}{2 \mu_{d}} \int \phi(\mathbf{r})(-\Delta) \phi(\mathbf{r}) d \mathbf{r}=\frac{1}{2 \mu_{d}} \int[\nabla \phi(\mathbf{r})]^{2} d \mathbf{r}
$$

is the familiar expression for the Coulomb energy in terms of the electric field $-\nabla \phi(\mathbf{r})$. Beyond some microscopic cutoff distance, the correlations of the electric potential in a

2 More general domain shapes were considered in refs. 5 and 6. 
Coulomb system are Gaussian ${ }^{(7)}$ and of the same form as the correlations associated with (1.3).

However the partition function of a Coulomb system is not identical to (1.3), in particular because (1.3) involves a functional integral on the potential rather than the familiar integral on the particle positions. A Gaussian transformation was made in ref. 1 for relating the Coulomb system to (1.3), but, although this has been useful for the slab geometry, we have not been able to deal with the disk geometry by this method.

We suggest here another approach, based on the heuristic assumption that the universal features of the grand partition function $\Xi_{C}$ of a conducting Coulomb system are correctly accounted for by the functional integral expression

$$
\Xi_{C}=\int \mathcal{D} \rho \exp \left[-\frac{\beta}{2} \int d \mathbf{r} d \mathbf{r}^{\prime} \rho(\mathbf{r}) G\left(\mathbf{r}, \mathbf{r}^{\prime}\right) \rho\left(\mathbf{r}^{\prime}\right)\right]
$$

where $\rho(\mathbf{r})$ is a charge density and $G\left(\mathbf{r}, \mathbf{r}^{\prime}\right)$ the Coulomb interaction solution of

$$
\Delta G\left(\mathbf{r}, \mathbf{r}^{\prime}\right)=-\mu_{d} \delta\left(\mathbf{r}-\mathbf{r}^{\prime}\right)
$$

with the appropriate (here Dirichlet) boundary conditions. In (2.2), the non-universal particle structure of the Coulomb system is already disregarded, and some cutoff prescription has to be made. Performing now a change of function from the charge density $\rho(\mathbf{r})$ to the electric potential $\phi(\mathbf{r})$, which are related to each other by

$$
\Delta \phi(\mathbf{r})=-\mu_{d} \rho(\mathbf{r})
$$

we obtain

$$
\Xi_{C}=\frac{D \rho}{D \phi} Z_{G}
$$

where $Z_{G}$ is the Gaussian partition function (1.3) and $D \rho / D \phi$ the Jacobian of the transformation from $\rho$ to $\phi$. Since

$$
Z_{G}=\left[\operatorname{det}\left(-\frac{1}{\mu_{d}} \Delta\right)\right]^{-\frac{1}{2}}
$$


and

$$
\frac{D \rho}{D \phi}=\operatorname{det}\left(-\frac{1}{\mu_{d}} \Delta\right)
$$

one obtains, from $(2.4), \Xi_{C}=1 / Z_{G}$ and

$$
\ln \Xi_{C}=-\ln Z_{G}
$$

Thus, $\ln \Xi_{C}$ and $\ln Z_{G}$ have the same universal term, except for its sign. The change of sign comes from the Jacobian $D \rho / D \phi$ in (2.4), i.e. the replacement of the charge density by the electric potential, as already explained in a different language in ref. 2 .

\section{SLAB}

We consider a slab in $d$ dimensions. Let us write the $d$-dimensional position vector as $\mathbf{r}=(\mathbf{x}, y)$, where $y$ is the $d$ th component and $\mathbf{x}$ stands for the $d-1$ other components. The Coulomb fluid fills the slab between $y=0$ and $y=W$, where there are ideal conductor walls.

In this Section, we derive the large- $W$ expansion (1.1) in general, in two different ways, and we check its validity on two two-dimensional solvable models. We make a comparison with the case of plain hard walls.

\subsection{Gaussian field theory route}

For the free energy of the two-dimensional Gaussian field theory in a strip, with Dirichlet boundary conditions, the expansion (1.1) with $C=-\pi / 24$ was derived in ref. 3 . The relation with Coulomb systems, discussed in Section 2, gives for Coulomb systems the expansion (1.1) with $C=\pi / 24$.

These considerations can be generalized to $d$ dimensions, for instance by adapting a method used in refs. 3 and 4, as follows. By considering the functional integral (1.3) as a path integral on an imaginary time interval $W$, one can express (1.3) in terms of the 
Hamiltonian of a quantum field theory in $d-1$ space dimensions. This Hamiltonian is a sum of terms

$$
H_{\mathbf{k}}=\frac{1}{2}\left(\frac{\mu_{d}}{\beta} \Pi_{\mathbf{k}}^{2}+\frac{\beta}{\mu_{d}} k^{2} \phi_{\mathbf{k}}^{2}\right)
$$

where $\mathbf{k}$ labels all possible wave-vectors in a space of dimension $d-1$ and $\Pi_{\mathbf{k}}$ is the canonical momentum conjugate to $\phi_{\mathbf{k}}$. Because of the Dirichlet boundary conditions $\phi=0$ at "times" 0 and $W,(1.3)$ is expressed in terms of $(0,0)$ matrix elements :

$$
Z_{G}=\prod_{\mathbf{k}}\left\langle\phi_{\mathbf{k}}=0\left|e^{-W H_{\mathbf{k}}}\right| \phi_{\mathbf{k}}=0\right\rangle=\prod_{\mathbf{k}}\left(\frac{\beta k}{2 \pi \mu_{d} \sinh W k}\right)^{1 / 2}
$$

Therefore

$$
\beta f=-\int \frac{d^{d-1} \mathbf{k}}{(2 \pi)^{d-1}} \ln \left(\frac{\beta k}{2 \pi \mu_{d} \sinh W k}\right)^{1 / 2} .
$$

For substracting off the ultraviolet divergences, it is convenient to consider first

$$
\beta \frac{\partial f}{\partial W}=\frac{1}{2} \int \frac{d^{d-1} \mathbf{k}}{(2 \pi)^{d-1}} k \operatorname{ctnh} W k
$$

and remove the $W=\infty$ value, which gives

$$
\begin{aligned}
\beta \frac{\partial f}{\partial W} & =\left.\beta \frac{\partial f}{\partial W}\right|_{W=\infty}+\frac{1}{2} \int \frac{d^{d-1} \mathbf{k}}{(2 \pi)^{d-1}} k(\operatorname{ctnh} W k-1) \\
& =\left.\beta \frac{\partial f}{\partial W}\right|_{W=\infty}+\frac{(d-1) \Gamma\left(\frac{d}{2}\right) \zeta(d)}{2^{d} \pi^{\frac{d}{2}} W^{d}}
\end{aligned}
$$

Thus, $\beta f$ has the universal finite-size correction $-C(d) / W^{d-1}$ for the Gaussian field theory, and (from Section 2) the correction is $C(d) / W^{d-1}$ for a Coulomb system, with $C(d)$ given by $(1.2)$.

\subsection{Screening sum rule route}

For the slab geometry, a more direct derivation of (1.1) is possible. It avoids any explicit reference to the Gaussian field theory, and therefore does not rely on the heuristic connexion of Section 2. Instead, this derivation uses a sum rule which expresses the perfect screening property of a conductor. 
Let us first consider a $d$-dimensional Coulomb system which fills the half-space $y>0$, with an ideal conductor wall at $y=0$. Let $\widehat{E}_{y}(0)$ be the microscopic electric field at some point, say the origin 0 , on the boundary and $\widehat{\rho}(\mathbf{r})$ the microscopic charge density at $\mathbf{r}=(\mathbf{x}, y)(y>0)$. The sum rule is

$$
\beta \int d \mathbf{r} y\left\langle\widehat{E}_{y}(0) \widehat{\rho}(\mathbf{r})\right\rangle^{T}=-1
$$

where $\langle\cdots\rangle^{T}$ means a truncated equilibrium statistical average : $\langle A B\rangle^{T}=\langle A B\rangle$ $-<A><B>$. One can derive ${ }^{(8)}$ (3.1) by considering the response of the system to an external infinitesimal point dipole $p$ oriented along the $y$-axis and located at the origin (on the Coulomb system side). This dipole adds to the Hamiltonian an interaction term $-p \widehat{E}_{y}(0)$, and by linear response theory the average charge density at $\mathbf{r}$ changes by $\delta \rho(\mathbf{r})=\beta p\left\langle\widehat{E}_{y}(0) \widehat{\rho}(\mathbf{r})\right\rangle^{T}$. Now, we assume that the Coulomb system has good screening properties : the induced charge density $\delta \rho(\mathbf{r})$ is localized near the origin and has a dipole moment which cancels $p$ :

$$
\int d \mathbf{r} y \delta \rho(\mathbf{r})=-p
$$

(since the dipole moment is defined with the origin chosen on the wall, there are no contributions of the surface charges on the ideal conductor wall). The sum rule (3.1) follows.

Let us now add a second ideal conductor wall at $y=W$. The good screening properties imply that, if $W$ is large, the local structure near the origin is unchanged, up to exponentially small effects. Therefore, the charge density $\delta \rho(\mathbf{r})$ is unchanged, its dipole moment (3.2) is unchanged (the integration range on $y$ in $(3.2)$ can be kept as $(0, \infty)$ since $\delta \rho(\mathbf{r})$ is localized near $y=0)$, and the sum rule (3.1) remains valid for the two-wall system.

This sum rule (3.1) can now be used for computing a universal correction to the pressure on the wall at $y=0$. In free $d$-dimensional space, the Coulomb interaction, solution of (2.3), is

$$
\begin{aligned}
& G_{0}\left(\mathbf{r}, \mathbf{r}^{\prime}\right)=-\ln \left(\left|\mathbf{r}-\mathbf{r}^{\prime}\right| / a\right) \quad, \quad d=2 \\
& G_{0}\left(\mathbf{r}, \mathbf{r}^{\prime}\right)=1 /\left|\mathbf{r}-\mathbf{r}^{\prime}\right|^{d-2}, \quad d>2
\end{aligned}
$$


( $a$ is some irrelevant length scale). With the present boundary conditions, the solution of (2.3) becomes

$$
\begin{gathered}
G\left(\mathbf{r}, \mathbf{r}^{\prime}\right)=G_{0}\left(\mathbf{r}-\mathbf{r}^{\prime}\right)-G_{0}\left(\mathbf{r}^{*}-\mathbf{r}^{\prime}\right)+\sum_{n=1}^{\infty}\left[G_{0}\left(\mathbf{r}+n 2 W \mathbf{u}-\mathbf{r}^{\prime}\right)\right. \\
\left.-G_{0}\left(\mathbf{r}^{*}+n 2 W \mathbf{u}-\mathbf{r}^{\prime}\right)+G_{0}\left(\mathbf{r}-n 2 W \mathbf{u}-\mathbf{r}^{\prime}\right)-G_{0}\left(\mathbf{r}^{*}-n 2 W \mathbf{u}-\mathbf{r}^{\prime}\right)\right]
\end{gathered}
$$

where $\mathbf{r}^{*}=(\mathbf{x},-y)$ is the image of $\mathbf{r}=(\mathbf{x}, y)$ with respect to the wall at $y=0$ and where $\mathbf{u}$ is the unit vector along the $y$ axis. Using

$$
\widehat{E}_{y}(0)=-\left.\int d \mathbf{r} \frac{\partial G\left(\mathbf{r}, \mathbf{r}^{\prime}\right)}{\partial y^{\prime}}\right|_{\mathbf{r}^{\prime}=0} \widehat{\rho}(\mathbf{r})
$$

we can write

$$
\left\langle\widehat{E}_{y}(0)^{2}\right\rangle^{T}=-\left.\int d \mathbf{r} \frac{\partial G\left(\mathbf{r}, \mathbf{r}^{\prime}\right)}{\partial y^{\prime}}\right|_{\mathbf{r}^{\prime}=0}\left\langle\widehat{E}_{y}(0) \widehat{\rho}(\mathbf{r})\right\rangle^{T} .
$$

Since $\left\langle\widehat{E}_{y}(0) \widehat{\rho}(\mathbf{r})\right\rangle^{T}$ is short-ranged, we can expand $\partial G / \partial y^{\prime}$ in powers of $W^{-1}$, at fixed $\mathbf{r}$, with the result

$$
\begin{aligned}
\left\langle\widehat{E}_{y}(0)^{2}\right\rangle^{T}=\left\langle\widehat{E}_{y}(0)^{2}\right\rangle_{W=\infty}^{T} & +\frac{\varepsilon(d-2)(d-1) \zeta(d)}{2^{d-2} W^{d}} \int d \mathbf{r} y\left\langle\widehat{E}_{y}(0) \widehat{\rho}(\mathbf{r})\right\rangle^{T} \\
& +O\left(1 / W^{d+1}\right)
\end{aligned}
$$

where $\varepsilon(d-2)=d-2$ if $d>2$ and $\varepsilon(d-2)=1$ if $d=2$. Let us describe the short-range interaction between the wall at $y=0$ and the particles by some potential $V(y)$, and let $n(y)$ be the particle number density. The pressure is

$$
p=-\int_{0}^{\infty} \frac{d V}{d y} n(y) d y-\frac{1}{2 \mu_{d}}\left\langle\widehat{E}_{y}(0)^{2}\right\rangle
$$

where the last term is the electrostatic pressure [if the short-range interaction is an infinite barrier at $y=y_{0},-d V / d y$ must be replaced by $\left.\beta^{-1} \delta\left(y-y_{0}\right)\right]$. Since the screening effect 
makes the density profiles near the wall $y=0$ independent of $W$ (up to exponentially small corrections), the related average field

$$
\left\langle\widehat{E}_{y}(0)\right\rangle=-\mu_{d} \int_{0}^{\infty}\langle\widehat{\rho}(y)\rangle d y
$$

is also independent of $W$. Thus, the pressure $(3.5)$, where $\left\langle\widehat{E}_{y}(0)^{2}\right\rangle$ can be rewritten as $\left\langle\widehat{E}_{y}(0)^{2}\right\rangle^{T}+\left\langle\widehat{E}_{y}(0)\right\rangle^{2}$, depends on $W$ only through (3.4). Using the sum rule (3.1) in (3.4) gives for the pressure $p$

$$
\beta p=\beta p(W=\infty)+\frac{(d-1) \Gamma\left(\frac{d}{2}\right) \zeta(d)}{2^{d} \pi^{\frac{d}{2}} W^{d}}+O\left(\frac{1}{W^{d+1}}\right)
$$

Since $\omega$, the grand potential per unit area, has the derivative

$$
\frac{\partial \omega}{\partial W}=-p
$$

the grand-potential expansion is indeed given by (1.1) and (1.2).

\subsection{The two-dimensional two-component plasma}

This is a two-dimensional system of particles of charges $\pm q$. The model is exactly solvable $^{(9,10)}$ at a special temperature $\beta^{-1}$ such that $\beta q^{2}=2$. For $\beta q^{2} \geq 2$, a pointparticle model is unstable against the collapse of pairs of oppositely charged particles ; we work with almost-point particles, introducing some short-distance cutoff in the interaction. In presence of ideal conductor walls, for $\beta q^{2} \geq 1$ the model would also be unstable against the collapse of a particle onto its electric image, unless some short-distance cutoff is also introduced in the wall-particle attraction.

In two dimensions, it is convenient to represent a position $\mathbf{r}=(x, y)$ by the complex coordinate $z=x+i y$, and the Coulomb interaction (3.3) can be explicitly summed into

$$
G\left(\mathbf{r}, \mathbf{r}^{\prime}\right)=-\ln \left|\frac{\sinh k\left(z-z^{\prime}\right)}{\sinh k\left(z-\bar{z}^{\prime}\right)}\right|
$$


where $k=\pi / 2 W$ (this result can also be obtained by a conformal transformation from the half-plane). The calculation for the present strip geometry is very similar to the one which has already been done ${ }^{(11)}$ for the half-plane case with only one ideal conductor wall. We start with a lattice model to avoid the collapse of positive and negative particles. There are two interwoven lattices $L_{+}$and $L_{-}$with $N_{+}$and $N_{-}$sites. The complex coordinates of the sites of $L_{+}\left(\operatorname{resp} . L_{-}\right)$are $\left\{u_{i}\right\}_{1 \leq i \leq N_{+}}\left(\right.$resp. $\left.\left\{v_{i}\right\}_{1 \leq i \leq N_{-}}\right)$. Positive particles can be on the sites of $L_{+}$and negative ones on those of $L_{-}$. Each site contains at most one particle. We work in the grand canonical ensemble and denote the fugacity by $\zeta$. The interaction between two particles is given by (3.7), and furthermore each particle has a self-interaction ${ }^{3}-\left(q^{2} / 2\right) \ln |a k / \sinh k(z-\bar{z})|$. Writing

$$
\sinh k\left(z-z^{\prime}\right)=\frac{1}{2} e^{-k\left(z+z^{\prime}\right)}\left(e^{2 k z}-e^{2 k z^{\prime}}\right)
$$

and using $\exp (2 k u)$ and $\exp (2 k v)$ instead of $u$ and $v$ in the Cauchy double alternant formula, we can follow the same steps as in ref.11 and we obtain for the grand partition function $\Xi=\operatorname{det}(1+K)$ where $K$ is the matrix

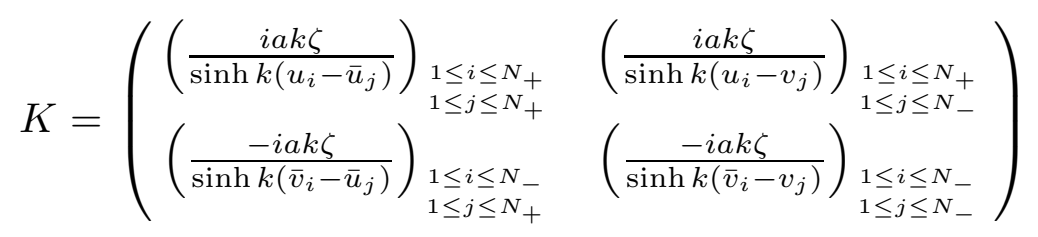

We now consider the continuum limit where the lattice spacing goes to zero. We define the rescaled fugacity $m=2 \pi a \zeta / S$ where $S$ is the area of a lattice cell. The eigenfunctions $(\psi, \chi)$ of $m^{-1} K$ and its eigenvalues $1 / \lambda$ are defined by the two coupled integral equations

$$
\begin{aligned}
& \frac{i \lambda}{4 W} \int_{D} d^{2} \mathbf{r}^{\prime}\left(\frac{1}{\sinh k\left(z-\bar{z}^{\prime}\right)} \psi\left(\mathbf{r}^{\prime}\right)+\frac{1}{\sinh k\left(z-z^{\prime}\right)} \chi\left(\mathbf{r}^{\prime}\right)\right)=\psi(\mathbf{r}) \\
& \frac{-i \lambda}{4 W} \int_{D} d^{2} \mathbf{r}^{\prime}\left(\frac{1}{\sinh k\left(\bar{z}-\bar{z}^{\prime}\right)} \psi\left(\mathbf{r}^{\prime}\right)+\frac{1}{\sinh k\left(\bar{z}-z^{\prime}\right)} \chi\left(\mathbf{r}^{\prime}\right)\right)=\chi(\mathbf{r})
\end{aligned}
$$

3 In equation (2.3c) of ref. 11 , exponents $1 / 2$ are missing for the first two factors on the r.h.s. 
where the domain of integration $D$ is the strip. In terms of these eigenvalues,

$$
\ln \Xi=\sum_{\lambda} \ln \left(1+\frac{m}{\lambda}\right)
$$

By using the equality

$$
\frac{\partial}{\partial \bar{z}} \frac{1}{\sinh k\left(z-z^{\prime}\right)}=\frac{\pi}{k} \delta\left(\mathbf{r}-\mathbf{r}^{\prime}\right), \quad \mathbf{r}, \mathbf{r}^{\prime} \in D
$$

we can transform the integral equations (3.7) into the differential equations

$$
2 \frac{\partial}{\partial \bar{z}} \psi=\lambda i \chi \quad \text { and } \quad 2 \frac{\partial}{\partial \bar{z}} i \chi=\lambda \psi
$$

In terms of the Dirac operator $\sigma \cdot \nabla$, these equations have the familiar ${ }^{(10)}$ form

$$
(\sigma \cdot \nabla) \Psi=\lambda \Psi
$$

where here $\Psi$ is the spinor $(\psi, i \chi)$. These equations can be combined into the Laplacian eigenvalue problem

$$
\Delta \psi=\lambda^{2} \psi
$$

with boundary conditions given by the integral equations (3.7):

$$
\psi(x, 0)=-\chi(x, 0) \quad \text { and } \quad \psi(x, W)=\chi(x, W)
$$

(incidentally, with such boundary conditions, $-\Delta$ turns out to be non-hermitian). We look for a solution of the form

$$
\psi(x, y)=e^{i v x}\left(A e^{i l y}+B e^{-i l y}\right) \quad \text { with } \quad l=i \sqrt{v^{2}+\lambda^{2}}
$$

The boundary conditions give two linear homogeneous equations for the coefficients $A$ and $B$. The determinant of this system must be zero for a non-vanishing solution to exist. This gives the relation between $v$ and $\lambda$ :

$$
\cosh \left(W \sqrt{v^{2}+\lambda^{2}}\right)-\lambda \frac{\sinh \left(W \sqrt{v^{2}+\lambda^{2}}\right)}{\sqrt{v^{2}+\lambda^{2}}}=0
$$


Let us define the entire fonction

$$
f(z)=\frac{1}{\cosh v W}\left[\cosh \left(W \sqrt{v^{2}+z^{2}}\right)-z \frac{\sinh \left(W \sqrt{v^{2}+z^{2}}\right)}{\sqrt{v^{2}+z^{2}}}\right]
$$

The solutions of (3.13) are the zeros of $f$ and we have $f(0)=1$, so

$$
f(z)=\prod_{\lambda \in f^{-1}(0)}\left(1-\frac{z}{\lambda}\right)
$$

Therefore, for each value of $v$, a partial summation of (3.9) can be made, giving for the grand potential $\omega$ per unit length

$$
\beta \omega=-\frac{1}{2 \pi} \int_{-\infty}^{+\infty} d v \ln \prod_{\lambda \in f^{-1}(0)}\left(1+\frac{m}{\lambda}\right)=-\frac{1}{\pi} \int_{0}^{+\infty} d v \ln f(-m)
$$

Now, in the limit $W \rightarrow \infty$

$$
\ln f(-m) \sim W\left(\sqrt{v^{2}+m^{2}}-|v|\right)+\ln \left(1+\frac{m}{\sqrt{v^{2}+m^{2}}}\right)-\ln \left(1+e^{-2|v| W}\right)+O\left(e^{-m W}\right)
$$

The first term gives the bulk contribution to the grand potential, the second the surface term and the third the universal finite-size correction. The bulk and surface terms would diverge if we did not take into account the particle-particle and particle-wall short-range repulsion. We can do so, for a repulsion of range $\sigma$, by introducing a cutoff $v_{\max }=1 / \sigma$.

The final result for the grand potential per unit length can be written as

$$
\beta \omega=-\beta p_{b} W+2 \beta \gamma_{c}+\frac{\pi}{24 W}+O\left(e^{-m W}\right)
$$

where

$$
\beta p_{b}=\frac{m^{2}}{2 \pi}\left(\ln \frac{2}{m \sigma}+1\right)
$$

and

$$
\beta \gamma_{c}=-\frac{m}{2 \pi}\left(\ln \frac{2}{m \sigma}+1-\frac{\pi}{2}\right)
$$

The bulk pressure $p_{b}$ is essentially the same as the one found for an infinite system ${ }^{(10)}$, up to a slightly different cutoff prescription. The surface tension $\gamma_{c}$ for ideal conductor walls 
is given by (3.16). And, finally, we find the finite-size correction $C(2) / W=\pi / 24 W$, as expected.

In the argument of Section 3.2, it was assumed that the local structure near one wall is unchanged, up to exponentially small corrections, by the presence of another wall at a large distance $W$. This assumption can be explicitly verified on the present model, by a calculation of the one-body and many-body densities, by the same method as in some previous work $^{(10,12)}$. These densities can be expressed in terms of Green functions $g_{s s^{\prime}}\left(\mathbf{r}, \mathbf{r}^{\prime}\right)$ $\left(s, s^{\prime}= \pm 1\right)$. The one-body density of particles of $\operatorname{sign} s$ is $n_{s}(\mathbf{r})=m g_{s s}(\mathbf{r}, \mathbf{r})$, the twobody truncated densities are $n_{s s^{\prime}}^{(2) T}\left(\mathbf{r}, \mathbf{r}^{\prime}\right)=-m^{2} g_{s s^{\prime}}\left(\mathbf{r}, \mathbf{r}^{\prime}\right) g_{s^{\prime} s}\left(\mathbf{r}^{\prime}, \mathbf{r}\right)$, etc... The matrix $g$ is defined by $g=m^{-1} K(1+K)^{-1}$, and therefore $g_{++}$and $g_{-+}$satisfy the integral equations

$$
\begin{array}{r}
g_{++}\left(\mathbf{r}_{1}, \mathbf{r}_{2}\right)+\frac{i m}{4 W} \int_{D} d^{2} \mathbf{r}\left(\frac{1}{\sinh k\left(z_{1}-\bar{z}\right)} g_{++}\left(\mathbf{r}, \mathbf{r}_{2}\right)+\frac{1}{\sinh k\left(z_{1}-z\right)} g_{-+}\left(\mathbf{r}, \mathbf{r}_{2}\right)\right) \\
=\frac{i}{4 W \sinh k\left(z_{1}-\bar{z}_{2}\right)} \\
g_{-+}\left(\mathbf{r}_{1}, \mathbf{r}_{2}\right)-\frac{i m}{4 W} \int_{D} d^{2} \mathbf{r}\left(\frac{1}{\sinh k\left(\bar{z}_{1}-\bar{z}\right)} g_{++}\left(\mathbf{r}, \mathbf{r}_{2}\right)+\frac{1}{\sinh k\left(\bar{z}_{1}-z\right)} g_{-+}\left(\mathbf{r}, \mathbf{r}_{2}\right)\right) \\
=\frac{-i}{4 W \sinh k\left(\bar{z}_{1}-\bar{z}_{2}\right)}
\end{array}
$$

(similar equations hold for $g_{--}$and $g_{+-}$). Differentiating these equations with respect to $\bar{z}_{1}$ and $z_{1}$ respectively, we find differential equations which can be combined into ${ }^{4}$

$$
\begin{gathered}
\left(m^{2}-\Delta_{1}\right) g_{++}\left(\mathbf{r}_{1}, \mathbf{r}_{2}\right)=m \delta\left(\mathbf{r}_{1}-\mathbf{r}_{2}\right) \\
g_{-+}\left(\mathbf{r}_{1}, \mathbf{r}_{2}\right)=\frac{i}{m}\left(\frac{\partial}{\partial x_{1}}+i \frac{\partial}{\partial y_{1}}\right) g_{++}\left(\mathbf{r}_{1}, \mathbf{r}_{2}\right)
\end{gathered}
$$

4 Refs. 10 and 11 use slightly different representations of the operator $K$. In the present section, following ref.11 rather than ref. 10 , we have in eq. (3.10) a spinor $\Psi=(\psi, i \chi)$ rather than $(\psi, \chi)$ and now a $g_{-+}$which is $i$ times the $g_{-+}$of ref. 10. This has no incidence on the physical quantities. 
Again the boundary conditions are given by the integral equations :

$$
g_{++}\left(x_{1}, 0 ; \mathbf{r}_{2}\right)=-g_{-+}\left(x_{1}, 0 ; \mathbf{r}_{2}\right) \quad \text { and } \quad g_{++}\left(x_{1}, W ; \mathbf{r}_{2}\right)=g_{-+}\left(x_{1}, W ; \mathbf{r}_{2}\right)
$$

For the present geometry it is useful to work with the Fourier transforms defined by

$$
g\left(\mathbf{r}_{1}, \mathbf{r}_{2}\right)=\int_{-\infty}^{+\infty} \frac{d l}{2 \pi} \hat{g}\left(y_{1}, y_{2}, l\right) e^{i l\left(x_{1}-x_{2}\right)}
$$

The solution for $\hat{g}_{++}$is:

$$
\begin{gathered}
\hat{g}_{++}\left(y_{1}, y_{2}, l\right)=\frac{m}{2 \kappa} e^{-\kappa\left|y_{1}-y_{2}\right|}+ \\
{\left[\frac{l}{2 \kappa}\left((\kappa+l) e^{\kappa\left(y_{1}+y_{2}\right)-\kappa W}-(\kappa-l) e^{-\kappa\left(y_{1}+y_{2}\right)+\kappa W}\right)-\frac{m}{\kappa}(\kappa-m) e^{-\kappa W} \cosh \kappa\left(y_{1}-y_{2}\right)\right]} \\
\times\left[(\kappa-m) e^{-\kappa W}+(\kappa+m) e^{\kappa W}\right]^{-1}
\end{gathered}
$$

with $\kappa=\sqrt{l^{2}+m^{2}}$. As $W \rightarrow+\infty$ for fixed values of $y_{1}$ and $y_{2}$, one finds after some manipulations

$$
\hat{g}_{++}\left(y_{1}, y_{2}, l\right)=\frac{m}{2 \kappa}\left(\exp \left[-\kappa\left|y_{1}-y_{2}\right|\right]+\frac{\kappa-l-m}{\kappa+l+m} \exp \left[-\kappa\left(y_{1}+y_{2}\right)\right]\right)+O\left(e^{-m W}\right)
$$

Up to an exponentially small term, this expression is the same one as in the case of one ideal conductor wall (see eq. (3.24) of ref.10). There are no algebraic correction in $1 / W$. This confirms our basic assumption of Section 3.2 that the correlation functions are unchanged, up to exponentially small effects, by the presence of the second wall.

\subsection{The two-dimensional one-component plasma}

This is a Coulomb system with only one species of mobile particles, of charge $q$, embedded in a background carrying a uniform charge density of the opposite sign. In two dimensions, the model is exactly solvable ${ }^{(13,14)}$ when $\beta q^{2}=2$. The half-plane geometry, with only one ideal conductor wall, has already been studied ${ }^{(15)}$. Since the particles repel each other, there is no pair collapse and one can deal with point-particles. In presence 
of ideal conductor walls however, one should prevent the collapse of a particle onto its electric image ; here we shall assume that an infinite potential barrier keeps the particles away from the walls by a distance $\varepsilon$, while the background extends up to the walls (this is slightly different from what has been assumed in ref. 15).

For the present strip geometry, the simplest approach is to take an appropriate limit of the two-component case. Instead of one fugacity, one can introduce different fugacities for positive and negative particles, and make the negative particle fugacity go to zero. Furthermore, a uniform background of charge density $-q \eta$ generates an electric potential $-\pi q \eta y(W-y)$ (it has the correct Laplacian $2 \pi q \eta$ and it vanishes on the walls at $y=0$ and $y=W)$; this potential can be taken into account by replacing the constant fugacity $\zeta$ of the positive particles by a position-dependent fugacity $\zeta \exp [2 \pi \eta y(W-y)]\left(\right.$ here $\left.\beta q^{2}=2\right)$, and adding to the grand potential the background self-energy which is $(1 / 6) \beta^{-1} \pi \eta^{2} W^{3}$ per unit length. Then, the set of equations (3.8) reduces to one equation

$$
\frac{i \lambda}{4 W} \int_{D} d^{2} \mathbf{r}^{\prime} \frac{e^{2 \pi \eta y^{\prime}\left(W-y^{\prime}\right)}}{\sinh \frac{\pi}{2 W}\left(z-\bar{z}^{\prime}\right)} \psi\left(\mathbf{r}^{\prime}\right)=\psi(\mathbf{r})
$$

where $D$ now is the strip between $y=\varepsilon$ and $y=W-\varepsilon$.

Since $z-\bar{z}^{\prime}$ does not vanish if $\mathbf{r}, \mathbf{r}^{\prime} \in D,(3.17)$ indicates that $\psi(\mathbf{r})$ is an analytical function of $z$. Because of the translational invariance along the $x$-axis, $\psi$ must depend on $x$ through a factor $\exp (i v x)$, and therefore $\psi=\exp (i v z)$. Using this $\psi$ in (3.17) and performing the integral upon $x^{\prime}$ gives the relation between $v$ and $\lambda$

$$
\frac{\lambda}{e^{2 W v}+1} \int_{\epsilon}^{W-\epsilon} e^{2\left[\pi \eta y^{\prime}\left(W-y^{\prime}\right)+v y^{\prime}\right]} d y^{\prime}=1
$$

Using this $\lambda$ in (3.9) (with the background self-energy added), performing the integral upon $y^{\prime}$, and making the change of variable $v=2 \pi \eta W t$, gives for the grand potential

$$
\begin{aligned}
-\beta \omega & =-\frac{\pi \eta^{2}}{6} W^{3} \\
& +2 \eta W \int_{0}^{+\infty} d t \ln \left\{1+\frac{m}{2 \sqrt{2 \eta}} \frac{e^{\frac{\pi}{2} W^{2} \eta\left(1+4 t^{2}\right)}}{2 \cosh \left(2 \pi \eta W^{2} t\right)}\left[\operatorname{erf}\left(\sqrt{2 \pi \eta} W\left(\frac{1}{2}-t-\frac{\epsilon}{W}\right)\right)\right.\right. \\
& \left.\left.+\operatorname{erf}\left(\sqrt{2 \pi \eta} W\left(\frac{1}{2}+t-\frac{\epsilon}{W}\right)\right)\right]\right\}
\end{aligned}
$$


where erf is the error function. Now we split the integral over $t$ into an integral going from 0 to $1 / 2$ and another one going from $1 / 2$ to $+\infty$. We write

$$
-\frac{\pi \eta^{2} W^{3}}{6}=2 \eta W \int_{0}^{1 / 2} \ln e^{-\pi \eta W^{2}(1-2 t)^{2} / 2} d t
$$

and add this term to the first integral. The resulting integral from 0 to $1 / 2$ can be separated in three terms which give, in the limit $W \rightarrow+\infty$, up to exponentially small terms, the bulk pressure, a first contribution for the surface tension and the universal finite-size correction

$$
\begin{gathered}
\beta p_{b} W=2 W \eta \int_{0}^{1 / 2} d t \ln \frac{m}{\sqrt{2 \eta}}=\eta W \ln \frac{m}{\sqrt{2 \eta}} \\
-2 \beta \gamma_{c}^{(1)}=2 W \eta \int_{0}^{1 / 2} d t \ln \left[\frac{\sqrt{2 \eta}}{m} e^{-\frac{\pi}{2} \eta W^{2}(1-2 t)^{2}}+\right. \\
\left.\frac{\operatorname{erf}\left(\sqrt{2 \pi \eta} W\left(\frac{1}{2}-t-\frac{\epsilon}{W}\right)\right)+\operatorname{erf}\left(\sqrt{2 \pi \eta} W\left(\frac{1}{2}+t-\frac{\epsilon}{W}\right)\right)}{2}\right] d t \\
=\sqrt{\frac{2 \eta}{\pi}} \int_{0}^{+\infty} d t \ln \left[e^{-t^{2}} \frac{\sqrt{2 \eta}}{m}+\frac{1}{2}(1+\operatorname{erf}(t-\sqrt{2 \pi \eta} \epsilon))\right] d t+O\left(e^{-m W}\right) \\
-\frac{\pi}{24 W}=-2 W \eta \int_{0}^{+\infty} d t \ln \left(1+e^{-4 \pi \eta W^{2} t}\right)+O\left(e^{-m W}\right)
\end{gathered}
$$

The integral from $1 / 2$ to $+\infty$ contributes only to the surface tension; it can be reexpressed as

$$
-2 \beta \gamma_{c}^{(2)}=\sqrt{\frac{2 \eta}{\pi}} \int_{0}^{+\infty} d t \ln \left[1+\frac{m}{2 \sqrt{2 \eta}} e^{t^{2}}(1-\operatorname{erf}(t+\epsilon \sqrt{2 \pi \eta}))\right]+O\left(e^{-m W}\right)
$$

The final result for the grand potential is

$$
\beta \omega=-\beta p_{b} W+2 \beta\left(\gamma_{c}^{(1)}+\gamma_{c}^{(2)}\right)+\frac{\pi}{24 W}+O\left(e^{-m W}\right)
$$

\subsection{Strip with plain hard walls}

The solvable two-dimensional models can also be used for studying the case of a strip with plain hard walls. The walls confine the particles, but there is no Dirichlet boundary condition for the electric potential, which freely "leaks out". The Coulomb interaction is 
just the free space one $-\ln \left(\left|\mathbf{r}-\mathbf{r}^{\prime}\right| / a\right)$. In that case, there is no finite-size correction of order $1 / W$ in the grand potential or free energy per unit length of the strip, as shown hereafter.

For the two-component plasma, the grand partition function is still given by (3.9) and (3.11), but the boundary conditions now are ${ }^{(2)}$

- on the boundary $y=0, \psi=g_{0}, \partial \psi / \partial \bar{z}=\bar{h}_{0}$

- on the boundary $y=W, \psi=g_{W}, \partial \psi / \partial \bar{z}=\bar{h}_{W}$

where $g_{0}, h_{0}$ (resp. $g_{W}, h_{W}$ ) are analytical functions in the domain $y<0$ (resp. $y>W$ ) vanishing at infinity. For $\psi$ of the form (3.12), these boundary conditions become

$$
\begin{array}{cll}
\psi(x, 0)=0 \quad, \quad \frac{\partial \psi}{\partial \bar{z}}(x, W)=0 & (v>0) \\
\psi(x, W)=0 \quad, \quad \frac{\partial \psi}{\partial \bar{z}}(x, 0)=0 & (v<0) .
\end{array}
$$

For each value of $v$, these equations give two linear homogeneous equations for the coefficients $A$ and $B$, the compatibility of which leads to the relation

$$
\cosh \left(W \sqrt{v^{2}+\lambda^{2}}\right)+|v| \frac{\sinh \left(W \sqrt{v^{2}+\lambda^{2}}\right)}{\sqrt{v^{2}+\lambda^{2}}}=0
$$

instead of (3.13). Now

$$
f(z)=e^{-W|v|}\left[\cosh \left(W \sqrt{v^{2}+z^{2}}\right)+|v| \frac{\sinh \left(W \sqrt{v^{2}+z^{2}}\right)}{\sqrt{v^{2}+z^{2}}}\right]
$$

and, in the limit $W \rightarrow \infty$,

$$
\left.\ln f(-m) \sim W\left(\sqrt{v^{2}+m^{2}}\right)-|v|\right)+\ln \left[\frac{1}{2}\left(1+\frac{|v|}{\sqrt{v^{2}+m^{2}}}\right)\right]+O\left(e^{-m W}\right)
$$

which gives for the grand potential $\omega$ per unit length

$$
\beta \omega=-\beta p_{b} W+2 \beta \gamma+O\left(e^{-m W}\right)
$$


The bulk pressure $p_{b}$ is the same as in (3.15), as expected. The surface tension $\gamma$ is now given by

$$
\beta \gamma=\frac{m}{2 \pi}\left(\frac{\pi}{2}-1\right)
$$

(an already known result ${ }^{(10)}$ ). And there is no finite-size correction, except for an exponentially small one.

For the one-component plasma in a strip with plain hard walls, the canonical free energy has been computed in ref. 16. In our notation, with the de Broglie wavelength and the length $a$ in the logarithmic potential taken as unity, the result for the free energy $f$ per unit length is

$$
\beta f=W \frac{\eta}{2} \ln \frac{\eta}{2 \pi^{2}}-2 \sqrt{\frac{\eta}{2 \pi}} \int_{0}^{Y} d t \ln \frac{\operatorname{erf}(Y+t)+\operatorname{erf}(Y-t)}{2}
$$

where $Y=W(\pi \eta / 2)^{1 / 2}$ and erf is the error function. In the large- $W$ limit one finds

$$
\beta f \sim W \frac{\eta}{2} \ln \frac{\eta}{2 \pi^{2}}-2 \sqrt{\frac{\eta}{2 \pi}} \int_{0}^{\infty} d t \ln \frac{1+\operatorname{erf}(t)}{2}+\frac{e^{-\pi \eta W^{2}}}{4 \pi^{2} \eta^{1 / 2} W^{2}}+\cdots
$$

The first term corresponds to the bulk free energy, the second one to the surface tensions ${ }^{(17)}$ on both boundaries, and the next term is indeed more than exponentially small ; there is no algebraic finite-size correction.

\section{DISK}

This Section is about a two-dimensional case : a Coulomb system in a disk of radius $R$, with an ideal conductor boundary. We derive the expansion (1.4) in general, and check it on two solvable models.

\subsection{General derivation}

We have not been able to make a direct derivation of (1.4) using, for instance, screening properties of the Coulomb system. We can only rely on the heuristic method of Section 
2, leading to the relation (2.5) between the Coulomb system and the Gaussian field theory. For the latter, it has been shown ${ }^{(5,6,18)}$ that $-\ln Z_{G}$ has a universal finite-size term -(1/6) ln $R$. From (2.5), we obtain (1.4).

\subsection{The two-component plasma}

The two-component plasma model, at $\Gamma=2$, is also solvable in the present geometry. In terms of complex coordinates $z=r e^{i \theta}$, with the origin at the center $O$ of the disk, the Coulomb potential solution of (2.3) with Dirichlet boundary conditions is obtained by the method of images as

$$
G\left(\mathbf{r}, \mathbf{r}^{\prime}\right)=-\ln \left|\frac{R\left(z-z^{\prime}\right)}{z \bar{z}^{\prime}-R^{2}}\right|
$$

Like in Section 3.3, we obtain (3.9) for the grand partition function. Now the sum is on the eigenvalues $\lambda$ defined by the two coupled integral equations

$$
\begin{aligned}
& \frac{\lambda}{2 \pi} \int_{D} d^{2} \mathbf{r}^{\prime}\left(\frac{-R}{z \bar{z}^{\prime}-R^{2}} \psi\left(\mathbf{r}^{\prime}\right)+\frac{1}{z-z^{\prime}} \chi\left(\mathbf{r}^{\prime}\right)\right)=\psi(\mathbf{r}) \\
& \frac{\lambda}{2 \pi} \int_{D} d^{2} \mathbf{r}^{\prime}\left(\frac{1}{\bar{z}-\bar{z}^{\prime}} \psi\left(\mathbf{r}^{\prime}\right)+\frac{-R}{\bar{z} z^{\prime}-R^{2}} \chi\left(\mathbf{r}^{\prime}\right)\right)=\chi(\mathbf{r})
\end{aligned}
$$

where the domain of integration $D$ is the disk. Using the equality

$$
\frac{\partial}{\partial \bar{z}} \frac{1}{z-z^{\prime}}=\pi \delta\left(\mathbf{r}-\mathbf{r}^{\prime}\right)
$$

we again find the Laplacian equation (3.11) with now the boundary condition $\chi=e^{i \theta} \psi$, i.e.

$$
\frac{1}{\lambda} e^{i \theta}\left(\frac{\partial}{\partial r}+\frac{i}{r} \frac{\partial}{\partial \theta}\right) \psi(r, \theta)-\left.e^{i \theta} \psi(r, \theta)\right|_{r=R}=0
$$

(again, $-\Delta$ is non-hermitian with that boundary condition).

Because of the circular symmetry, we look for eigenfunctions of the form

$$
\psi=I_{\ell}(\lambda r) e^{i \ell \theta} \quad, \quad \ell \in \mathbb{Z}
$$


where $I_{\ell}$ is a modified Bessel function. The boundary condition (4.2) imposes

$$
I_{\ell}^{\prime}(\lambda R)-\frac{\ell}{\lambda R} I_{\ell}(\lambda R)-I_{\ell}(\lambda R)=0
$$

i.e.

$$
I_{\ell+1}(\lambda R)-I_{\ell}(\lambda R)=0
$$

All the eigenvalues $\lambda$ are obtained by taking, for each value of $\ell \in \mathbb{Z}$, all the non-zero roots of (4.3). Since (4.3) is invariant under the transformation $\ell \rightarrow-\ell-1$, it is enough to consider the eigenvalues $\lambda_{\ell}$ associated to $\ell \in I N$, counting each of them twice. Then, as in Section 3.3, for each value of $\ell$ a partial summation of (3.9) can be made by noting that the corrresponding eigenvalues $\lambda_{\ell}$ are the zeros of the function $f_{\ell}(z)$ :

$$
f_{\ell}(z)=\ell !\left(\frac{2}{z R}\right)^{\ell}\left[I_{\ell}(z R)-I_{\ell+1}(z R)\right], \quad \ell \geq 0
$$

The prefactor in (4.4) has been chosen in such a way that $f_{\ell}(0)=1$. Therefore, the entire function $f_{\ell}(z)$ can be written as the product

$$
f_{\ell}(z)=\prod_{\lambda_{\ell}}\left(1-\frac{z}{\lambda_{\ell}}\right)
$$

and

$$
\sum_{\lambda_{\ell}} \ln \left(1+\frac{m}{\lambda_{\ell}}\right)=\ln \prod_{\lambda_{\ell}}\left(1+\frac{m}{\lambda_{\ell}}\right)=\ln f_{\ell}(-m)
$$

making (3.9) a sum on $\ell$ only :

$$
\ln \Xi=2 \sum_{\ell=0}^{\infty} \ln \left\{\ell !\left(\frac{2}{m R}\right)^{\ell}\left[I_{\ell}(m R)+I_{\ell+1}(m R)\right]\right\} .
$$

For obtaining a large- $R$ asymptotic expansion of (4.5), one can use the Debye expansion ${ }^{(19)}$ of $I_{\ell}(m R)$. This expansion is the one which is appropriate here, since both $m R$ and $\ell$ become large. It is convenient to split (4.5) as

$$
\ln \Xi=S_{1}+S_{2}
$$


where

$$
S_{1}=2 \sum_{\ell=0}^{\infty} \ln \left[\ell !\left(\frac{2}{m R}\right)^{\ell} I_{\ell}(m R)\right]
$$

and

$$
S_{2}=2 \sum_{\ell=0}^{\infty} \ln \left[1+\frac{I_{\ell+1}(m R)}{I_{\ell}(m R)}\right]
$$

since $S_{1}$ is $\ln \Xi$ for a plain hard wall boundary and has already been evaluated in ref. 2 . As to $S_{2}$, rewritten and cut off as

$$
S_{2}=2 \sum_{\ell=0}^{L} \ln \left[1+\frac{I_{\ell}^{\prime}(m R)}{I_{\ell}(m R)}-\frac{\ell}{m R}\right]
$$

it can be evaluated by similar techniques, using the Debye expansion of $I_{\ell}$ and $I_{\ell}^{\prime}$, and using for the sum on $\ell$ the Euler-MacLaurin summation formula . If we now take into account the particle-particle and particle-wall short range repulsions by choosing the cutoff as $L=R / \sigma$, we find

$$
S_{2}=m R \ln \frac{2}{m \sigma}+\frac{1}{2} \ln 2-\frac{\pi}{4}+O\left(\frac{1}{m R}\right)
$$

In (4.6), all terms which vanish as the cutoff $\rightarrow \infty$ have already been removed.

Adding $S_{1}$ (from ref. 2) and $S_{2}$, one finds for the grand potential $\Omega$, in the case of an ideal conductor boundary,

$$
\beta \Omega=-\ln \Xi=-\beta p_{b} \pi R^{2}+\beta \gamma_{c} 2 \pi R+\frac{1}{6} \ln m R-\frac{5}{12}-\frac{1}{6} \ln 2-2 \zeta^{\prime}(-1)+\frac{\pi}{4}
$$

where the bulk pressure $p_{b}$ and the surface tension $\gamma_{c}$ are, as expected, given by the same expressions (3.15) and (3.16) as in the strip geometry. And (4.7) has the expected universal finite-size correction $(1 / 6) \ell n R$.

One should note that there is no $\ln R$ term in $S_{2}$; it comes entirely from $S_{1}$. In other words, the universal finite-size correction is the same one for a plain hard wall and for an ideal conductor wall, unlike in the strip geometry. 


\subsection{The one-component plasma}

We consider the same model as in Section 3.4, but in the disk geometry. The electric potential generated by the background is $-\frac{1}{2} \pi \eta q\left(R^{2}-r^{2}\right)$. The background self-energy adds to the grand potential a term $\frac{1}{4} \beta^{-1}\left(\pi \eta R^{2}\right)^{2}$ The eigenvalue integral equation now is

$$
\frac{-\lambda R}{2 \pi} \int_{D} \frac{\exp \left[\pi \eta\left(R^{2}-r^{2}\right)\right]}{\bar{z}^{\prime} z-R^{2}} \psi\left(\mathbf{r}^{\prime}\right) d^{2} \mathbf{r}^{\prime}=\psi(\mathbf{r})
$$

Again we notice that $\psi$ is analytical. Because of the rotational invariance, $\psi$ must depend on $\theta$ through a factor $\exp (i \ell \theta)$, and therefore $\psi(\mathbf{r})=z^{\ell}$ with $\ell$ a non-negative integer. Using this form in (4.8) we have the relation between $\lambda$ and $\ell$

$$
\lambda \exp \left[\pi \eta R^{2}\right] \int_{0}^{R-\epsilon}\left(\frac{r}{R}\right)^{2 \ell+1} \exp \left[-\pi \eta r^{2}\right] d r=1
$$

The grand potential becomes, after some manipulations,

$$
\begin{aligned}
\beta \Omega & =\frac{1}{4}\left(\pi \eta R^{2}\right)^{2} \\
& -\sum_{\ell=0}^{+\infty} \ln \left[1+\frac{m R \exp \left(\pi \eta R^{2}\right)}{2\left(\pi \eta R^{2}\right)^{\ell+1}} \gamma\left(\ell+1, \pi \eta(R-\epsilon)^{2}\right)\right]
\end{aligned}
$$

where $\gamma(n, x)=\int_{0}^{x} t^{n-1} \exp (-t) d t$ is the incomplete gamma function. Let $N=\pi \eta R^{2}$. We split the sum over $\ell$ in eq. (4.9) into a sum $S_{1}$ for $0 \leq \ell \leq N$ and a sum $S_{2}$ for $\ell>N$. Using

$$
e^{N} N^{-(\ell+1)} \ell ! \sim \sqrt{\frac{2 \pi}{N}} \exp \left[\frac{(\ell-N)^{2}}{2 N}+\frac{\ell-N}{2 N}\right]
$$

we have in the limit $R \rightarrow+\infty$

$$
\begin{aligned}
S_{1} & =(N+1) \ln \left(\frac{m R}{2}\right) \\
& +\sum_{\ell=0}^{N}[-(\ell+1) \ln N+N+\ln \ell !] \\
& +\sum_{\ell=0}^{N} \ln \left[\frac{\sqrt{2 \eta}}{m} e^{-\frac{(\ell-N)^{2}}{2 N}}+\frac{1}{\ell !} \gamma\left(\ell+1, \pi \eta(R-\epsilon)^{2}\right)\right]
\end{aligned}
$$


Using Stirling's formula, the asymptotic formulas for $R \rightarrow+\infty$

$$
\begin{aligned}
\sum_{\ell=0}^{N} \ln \ell ! & =(N+1) \ln N !-\frac{N^{2}}{2} \ln N \\
& +\frac{N^{2}}{4}-\frac{1}{2} N \ln N-\frac{1}{12} \ln N+O(1)
\end{aligned}
$$

and

$$
\frac{1}{\ell !} \gamma\left(\ell+1, \pi \eta(R-\epsilon)^{2}\right)=\frac{1}{2}\left[1+\operatorname{erf}\left(\frac{N-\ell}{\sqrt{2 N}}-\epsilon \sqrt{2 \pi \eta}\right)\right]+O\left(\frac{1}{\sqrt{N}}\right)
$$

we obtain, in the small $-\epsilon$ limit,

$$
\begin{aligned}
S_{1} & =-\frac{1}{4}\left(\pi \eta R^{2}\right)^{2}-\pi \eta R^{2} \ln \left(\frac{m}{\sqrt{2 \eta}}\right)+\frac{1}{6} \ln \left[(2 / m)^{6}(\pi \eta)^{7 / 2} R\right] \\
& +\sqrt{2 \pi \eta} R \int_{0}^{+\infty} d t \ln \left[\frac{\sqrt{2 \eta}}{m} e^{-t^{2}}+\frac{1}{2}(1+\operatorname{erf}(t-\epsilon \sqrt{2 \pi \text { eta }}))\right]+O(1)
\end{aligned}
$$

In $S_{2}$ we use (4.13) and (4.10) to find

$$
S_{2}=\sqrt{2 \pi \eta} R \int_{0}^{+\infty} d t \ln \left[1+\frac{m}{2 \sqrt{2 \eta}} e^{t^{2}}(1-\operatorname{erf}(t+\epsilon \sqrt{2 \pi \eta}))\right]
$$

Putting (4.14) and (4.15) in (4.9) we have

$$
\beta \Omega=-\beta p_{b} \pi R^{2}+2 \pi R \beta\left(\gamma_{c}^{(1)}+\gamma_{c}^{(2)}\right)+\frac{1}{6} \ln \left[\left(\frac{2}{m}\right)^{6}(\pi \eta)^{7 / 2} R\right]+O(1)
$$

where the bulk pressure $p_{b}$ is given by (3.18) and $\gamma_{c}^{(1)}$ and $\gamma_{c}^{(2)}$ are given by (3.19) and (3.20) in the small- $\epsilon$ limit. We find the universal finite-size correction $\frac{1}{6} \ln R$.

It is interesting to compare the surface tension for the one-component plasma in the limit $\epsilon \rightarrow 0$ and the surface tension for the two-component plasma in the limit $\sigma \rightarrow 0$. We have in that limit for the one-component plasma

$$
\gamma_{c}^{(2)} \sim \frac{m}{4 \pi} \ln \epsilon
$$

and for the two-component plasma

$$
\gamma_{c} \sim \frac{m}{2 \pi} \ln \sigma
$$


The surface tension of the one-component plasma is one-half the one of the two-component plasma.

\section{CONCLUSION}

The grand potential of a Coulomb system has universal finite-size corrections very similar to the ones which occur for a simple critical system : the massless Gaussian field theory. The boundary conditions however play an important rôle. In the geometry of a slab, if the boundaries are ideal conductor walls, a Coulomb system has a grand potential with a universal finite-size correction which is just the opposite of the one found for a Gaussian field with Dirichlet boundary conditions; but if the boundaries are plain hard walls, the Coulomb system no longer exhibits that finite-size correction. In the geometry of a disk, a two-dimensional Coulomb system has a finite-size correction to its grand potential which is the same one for an ideal conductor wall and for the previously studied ${ }^{(2)}$ case of a plain hard wall (in both cases, again the correction is opposite to the one occuring for the Gaussian field). An argument given in ref. 2 essentially says that a Coulomb system with plain hard walls looks somewhat like an ideal conductor when seen from the outside; this might be relevant for explaining that the curved boundary of a disk generates the same correction (1/6) $\ln R$ in both plain hard wall and ideal conductor wall cases. Nevertheless, a unified treatment of the different possible boundary conditions still has to be found.

In the case of a slab with ideal conductor walls, the finite-size correction to the grand potential of a Coulomb system was derived on the sole basis of the screening effect (Section 3.2). A more general derivation on the basis of the screening effect should be found for other geometries and boundary conditions. 


\section{$\underline{\text { References }}$}

1. J.P. Forrester, J. Stat. Phys. 63 : 491 (1991)

2. B. Jancovici, G. Manificat, and C. Pisani, J. Stat. Phys. 76 : 307 (1994)

3. H.W.J. Blöte, J.L. Cardy, and M. P. Nightingale, Phys. Rev. Lett. 56 : 742 (1986).

4. I. Affleck, Phys. Rev. Lett. 56 : 746 (1986).

5. J.L. Cardy and I. Peschel, Nucl. Phys. B 300 [FS 22] : 377 (1988).

6. J.L. Cardy, in Fields, Strings and Critical Phenomna, Les Houches 1988, E. Brézin and J. Zinn-Justin, eds. (North-Holland, Amsterdam, 1990).

7. B. Jancovici, J. Stat. Phys. 80 : (1995).

8. B. Jancovici, J. Phys. (France) $47: 389$ (1986).

9. M. Gaudin, J. Phys. (France) $46: 1027$ (1985).

10. F. Cornu and B. Jancovici, J. Chem. Phys. 90 : 2444 (1989).

11. P.J. Forrester, J. Chem. Phys. 95 : 4545 (1990).

12. B. Jancovici and G. Manificat, J. Stat. Phys. 68 : 1089 (1992).

13. A. Alastuey and B. Jancovici, J. Phys. (France) $42: 1$ (1981).

14. B. Jancovici, Phys. Rev. Lett. 46 : 386 (1981).

15. P.J. Forrester, J. Phys. A 18 : 1419 (1985).

16. P.J. Forrester and E.R. Smith, J. Phys. A 15 : 3861 (1982).

17. V. Russier, J.P. Badiali, and M.L. Rosinberg, J. Phys. C 18 : 707 (1985)

18. O. Alvarez, Nucl. Phys. B 216 : 125 (1983).

19. M. Abramowitz and I.A. Stegun, Handbook of Mathematical Functions (National Bureau of Standards, Washington D.C., 1964). 